\title{
Leptin: a novel therapeutic role in lipodystrophy
}

\author{
D.B. Savage and S. O’Rahilly \\ Address correspondence to: S. O'Rahilly, Departments of Clinical Biochemistry and Medicine, \\ University of Cambridge, Addenbrooke's Hospital, Box 157, Cambridge CB2 2QR, United Kingdom. \\ Phone: 44-1223-336855; Fax: 44-1223-330160; E-mail: sorahill@hgmp.mrc.ac.uk.
}

Departments of Clinical Biochemistry and Medicine, University of Cambridge, Addenbrooke's Hospital, Cambridge, United Kingdom

J. Clin. Invest. 109:1285-1286 (2002). doi:10.1172/JCI200215326.

Lipodystrophic syndromes encompass a heterogeneous group of rare disorders characterized by partial or generalized loss of adipose tissue depots (1). They are commonly associated with dyslipidemia, hepatic steatosis, and severe insulin resistance (2). The fact that insulin resistance and the consequent progression to diabetes can result from either obesity or lipodystrophy reflects the crucial role of adipose tissue in carbohydrate and lipid metabolism. In the absence of adequate adipocyte capacity, excess calories cannot be diverted to their normal storage depot; instead they accumulate as increased triglyceride stores in liver, in skeletal and cardiac muscle, and in the pancreatic $\beta$ cell. This extra-adipose lipid accumulation, through asyet unclear means, is associated with impaired insulin action and, often, diabetes.

In addition to their passive role as storage depots, normal adipocytes secrete a number of peptides ("adipokines") that may influence insulin sensitivity and/or energy balance $(3,4)$. These include potential insulin sensitizers, such as leptin and Acrp30 (also known as adiponectin), and insulin antagonists, including TNF- $\alpha$, IL- 6 , and possibly resistin. The insulin resistance of lipodystrophy may therefore be the result of disturbed lipid fluxes and/or abnormalities of adipokine secretion.

The striking similarity of murine models of lipodystrophy to the human phenotype has provided insights into the metabolic disarray observed in this disorder as well as revealing several therapeutic options. Insulin sensitivity improves dramatically following fat transplantation in mice with generalized lipodystrophy, a finding that was first taken to suggest a critical role for adipocyte mass per se (5). However, the failure of fat transplanted from $o b / o b$ (leptin-deficient) mice to reverse the metabolic disturbance highlighted the importance of adipocyte-derived leptin (6). Further support for this notion comes from the previously described beneficial effects of leptin administration in a different lipodystrophic strain of mice (7). More recently, Yamauchi et al. have suggested that combined leptin and adiponectin infusions are required to fully restore normal insulin action in yet another lipodystrophic mouse model (8).

Adipose tissue transplantation in humans has been considered but has not yet been reported. As an alternative to such surgery, might adipose mass be stimulated pharmacologically? The nuclear hormone receptor PPAR $\gamma$ plays a key role in adipogenesis, and its agonists increase fat mass in humans and appear to do the same in at least some lipodystrophic patients (9). However, the impact of this approach in generalized lipodystrophy has yet to be convincingly demonstrated. It may also confer some risk of increased hepatic steatosis, as is seen in lipodystrophic mice treated with rosiglitazone, a known PPAR $\gamma$ agonist (10).

\section{Leptin therapy in lipodystrophic patients}

An alternative to increasing adipocyte fat stores is to limit the caloric load on these stores by reducing nutrient intake. Recombinant leptin has been successfully used to ameliorate hyperphagia in leptindeficient obese humans (11). Since generalized lipodystrophy also leads to extreme hypoleptinemia and hyperphagia, Oral et al. wondered if this therapeutic approach might be of similar benefit in lipodystrophy. The results of their work are reported in two papers. Their first paper, which appears in a recent issue of the New England Journal of Medicine, describes dramatic metabolic improvements in nine patients treated with daily subcutaneous leptin injections for 4 months (12). Glycemic control, hypertriglyceridemia, and hepatic steatosis were strikingly improved despite significant reductions in the use of oral hypoglycemics and insulin therapy. As expected, self-reported caloric intake decreased. Somewhat surprisingly, although consistent with previous reports on the use of leptin in humans (11), resting metabolic rate also fell significantly.

In this issue of the JCI, Petersen et al. report detailed physiological studies in a subset of these patients (13). Hyperinsulinemic-euglycemic clamps were used to document both reduced peripheral glucose disposal and increased hepatic glucose output in three patients prior to commencing leptin therapy. Leptin therapy markedly improved both parameters. This improvement was associated with significant reductions in hepatic steatosis and intramyocellular triglycerides, providing a potential mechanism for the improved insulin sensitivity. However, as the authors acknowledge, it is still not clear whether triglycerides themselves interfere with insulinstimulated glucose uptake. In fact, base-line levels of intramyocellular triglycerides were similar in patients with generalized lipodystrophy and normal controls. The authors suggest that a fatty acid-derived intermediate may be inducing insulin 
resistance and provide data demonstrating a fall in muscle fatty acylCoA's in a skeletal muscle biopsy from one patient.

\section{Sites of leptin action}

Leptin clearly has dramatic effects on appetite in humans and rodents. What is less clear is whether it has additional peripheral antisteatotic effects in these species. Unger and coworkers accumulated a large body of evidence in rodents to support the hypothesis that leptin is an antisteatotic peptide (14), and Minokoshi et al. recently suggested that this effect might be mediated via AMP-activated protein kinase activation, with secondary induction of lipid oxidation (15). This action of leptin appeared to be mediated both centrally via the sympathetic nervous system and peripherally via the long form of the leptin receptor (Ob-Rb) (15). Whether this observation will translate to humans remains to be explored. Of note, in contrast to children with total lipodystrophy, severely obese children with complete, genetic leptin deficiency do not have radiologically detectable hepatic steatosis, nor are they as severely insulin-resistant (S. O'Rahilly and I.S. Farooqi, unpublished observations). This suggests, firstly, that the metabolic consequences of generalized lipodystrophy are unlikely to be entirely attributable to very low levels of circulating leptin, and secondly, that the clinical response to leptin therapy is likely to be partial at best.

Nevertheless, there is now no doubt that leptin therapy is of benefit. How might it work? In mice, it is clear that leptin has metabolic effects independent of food intake, as hypoleptinemic mice treated with leptin show greater improvements in weight and/or disordered metabolism than do pair-fed controls (16). In humans, the extent to which leptin improves insulin action through a suppression of appetite, rather than a direct or indirect effect on insulin-sensitive tissues, is still unclear. In part this reflects inherent difficulties in the accurate, long-term measurement of food intake in humans.

\section{Who might benefit \\ from leptin therapy?}

The discovery of leptin in 1994 raised hopes that it might represent a panacea for the huge public health problem of obesity. Subsequently, however, it was found that most obese people have relatively high levels of circulating leptin (17), and, while augmenting those levels pharmacologically has some effect on fat mass, the very limited clinical trial information thus far in the public domain suggests that these effects are neither dramatic nor consistent (18). This may relate to the possibility of a rather ill-defined state of leptin resistance existing in most obese humans, or perhaps more plausibly, to the evolution of leptin primarily to signal transitions between states of starvation and nutritional adequacy (19). Taking this latter view, it is not surprising that leptin therapy appears to be most dramatically effective in states where circulating leptin levels are markedly and inappropriately low for the true levels of energy stores. Thus, in congenital leptin deficiency, where the principal clinical abnormality is a massively expanded fat mass, the major therapeutic impact of leptin is to reduce that fat mass. In contrast, in generalized lipodystrophy, where excess fat cannot be stored in adipose tissue, leptin's major impact results from the reduction of excessive fat storage in nonadipose tissue with a marked amelioration of the resulting insulin resistance. Thus, we now know of two distinct human diseases where the correction of near aleptinemia is of major therapeutic benefit.

Could leptin be of benefit in more common conditions of relative hypoleptinemia? Farooqi et al. (20) recently reported that individuals heterozygous for nonsense mutations in the Leptin gene had a higher body fat content than would be predicted by their height and weight, accompanied by relatively low plasma levels of leptin. This observation provides impetus for therapeutic trials of leptin supplementation in subgroups of obese subjects with relatively low leptin levels. Similarly, syndromes of partial lipodystrophy, either genetic or acquired (1), are considerably more common than the complete form and are often accompanied by severe metabolic dysfunction. Trials of leptin therapy in these types of subjects will be illuminating.

1. Garg, A. 2000. Lipodystrophies. Am. J. Med. 108:143-152.

2. Reitman, M.L., Arioglu, E., Gavrilova, O., and Taylor, S.I. 2000. Lipoatrophy revisited. Trends Endocrinol. Metab. 11:410-416.

3. Kahn, B.B., and Flier, J.S. 2000. Obesity and insulin resistance. J. Clin. Invest. 106:473-481.

4. Steppan, C.M., and Lazar, M.A. 2002. Resistin and obesity-associated insulin resistance. Trends Endocrinol. Metab.13:18-23.

5. Gavrilova, O., et al. 2000. Surgical implantation of adipose tissue reverses diabetes in lipoatrophic mice. J. Clin. Invest.105:271-278.

6. Friedman, J. 2002. Fat in all the wrong places. Nature. 415:268-269.

7. Shimomura, I., Hammer, R.E., Ikemoto, S., Brown, M.S., and Goldstein, J.L. 1999. Leptin reverses insulin resistance and diabetes mellitus in mice with congenital lipodystrophy. Nature. 401:73-76.

8. Yamauchi, T., et al. 2001. The fat-derived hormone adiponectin reverses insulin resistance associated with both lipoatrophy and obesity. Nat. Med. 7:941-946.

9. Arioglu, E., et al. 2000. Efficacy and safety of troglitazone in the treatment of lipodystrophy syndromes. Ann. Intern. Med. 133:263-274.

10. Chao, L., et al. 2000. Adipose tissue is required for the antidiabetic, but not for the hypolipidemic, effect of thiazolidinediones. J. Clin. Invest. 106:1221-1228.

11. Farooqi, I.S., et al. 1999. Effects of recombinant leptin therapy in a child with congenital leptin deficiency. N. Engl. J. Med. 341:879-884.

12. Oral, E.A., et al. 2002. Leptin-replacement therapy for lipodystrophy. N. Engl.J. Med. 346:570-578.

13. Petersen, K.F., et al. 2002. Leptin reverses insulin resistance and hepatic steatosis in patients with severe lipodystrophy. J. Clin. Invest. 109:1345-1350. doi:10.1172/JCI200215001.

14. Unger, R.H. 2002. Lipotoxic diseases. Annu. Rev. Med. 53:319-336.

15. Minokoshi, Y., et al. 2002. Leptin stimulates fattyacid oxidation by activating AMP-activated protein kinase. Nature. 415:339-343.

16. Pelleymounter, M.A., et al. 1998. Efficacy of exogenous recombinant murine leptin in lean and obese 10- to 12 -mo-old female CD-1 mice. Am. J. Physiol. 275:R950-R959.

17. Considine, R.V., Sinha, M.K., Heiman, M.L., et al. 1996. Serum immunoreactive-leptin concentrations in normal-weight and obese humans. N. Engl. J. Med. 334:292-295.

18. Mantzoros, C.S., and Flier, J.S. 2000. Editorial Leptin as a therapeutic agent: trials and tribulations. J. Clin. Endocrinol. Metab. 85:4000-4002.

19. Ahima, R.S., et al. 1996. Role of leptin in the neuroendocrine response to fasting. Nature. 382:250-252.

20. Farooqi, I.S., Keogh, J.M., Kamath, S., et al. 2001 Partial leptin deficiency and human adiposity. Nature. 414:3-5. 